\title{
Agro Image De-Noising (Aid) for Enhanced Agricultural Images
}

\author{
Sangeetha Muthiah, A. Senthilrajan
}

\begin{abstract}
Several Noises may be present in acquired images. This is an undesired feature for image processing techniques that analyze these images. Image de-noising helps improve efficiency of image processing. Many image de-noising methods have been proposed and exist in literature. Image de-noising methods for agricultural images have been proposed to a lesser extent when compared to the bright medical or photographic images. This paper proposes Agricultural Image De-noising (AID) which uses a discrete wavelet transform (DWT) to eliminate noise in agricultural images. This study uses specific kind of wavelet family spline wavelet transforms with appropriate decomposition level and the wavelet coefficients are analysed with hard and soft threshold methods. The denoised image using various spline wavelets is compared of hard threshold and soft threshold are assessed. The performance of AID is calculated using the peak signal to noise ratio (PSNR) and signal to noise ratio (SNR).
\end{abstract}

Keywords: Agricultural Image De-noising, DWT, Spline wavelet

\section{INTRODUCTION}

India has a large agriculture sector expanding every year. Growth in agricultural production has a positive impact on the rural income and the livelihood of the people. Despite the increase in crop production, the climate change induced stress affects crop growth and reduces the yield. Another important factor caused by biotic pest which results in crop damage and revenue loss.

Thus, to protect the crops from pests farmers often turn to use pesticides to increase the yield and quality of the produce. The pesticides are meant to kill weeds and insects and has benefits in terms of increased food production and higher income. On the other side, it interrupts the eco-system by polluting soil, contaminating groundwater and can be deadly to other living organisms. In India $76 \%$ of the pesticide used is insecticide, as against $44 \%$ globally [1]. Therefore, the agriculture producers must gain knowledge about the timing of the pesticide application and must avoid applying the same pesticides at every stage of the crop development [2]. The rational and efficient use of pesticides can improve

Revised Manuscript Received on December 30, 2019.

* Correspondence Author

Sangeetha Muthiah*, Department of Cmputational Logistics, Alagappa University, Karaikudi (Tamil nadu) India.

E-mail: sangeethsaro@yahoo.com

Dr. A. Senthilrajan, Department of Cmputational Logistics, Alagappa University, Karaikudi (Tamil nadu) India. E-mail: agni_senthil@yahoo.com

(C) The Authors. Published by Blue Eyes Intelligence Engineering and Sciences Publication (BEIESP). This is an open access article under the CC BY-NC-ND license (http://creativecommons.org/licenses/by-nc-nd/4.0/) agricultural food production and reduce the negative impact on our eco-system. The farmers must adopt scientific strategies to obtain data related to pest prevention and control measures to limit the use of pesticides for precise management of crop disease.

The identification and management of disease in agricultural fields is widespread. The continuing challenge is early pathogen detection followed by effective control measures are necessary to prevent spread of pests and disorders in crops [3]. Therefore crop producers often turn to agricultural professionals for effective diagnosis which is a labour intensive and time consuming process. Hence, delayed diagnosis leads to spread of pests which cause crop damage and revenue loss. Digital Image Processing refers to processing images with the help of a computer. The digital image processing continues to expand over the years in many fields. It has vigorous growth and has been the subject of study and research in fields such as medical diagnosis, forensics, engineering, defense, agriculture and scientific research. The structure of Digital image processing comprises image acquisition, image processing and image analysis. The human eye working principle is analogous to the optical system of a common digital camera, since the incoming light passes through an aperture (pupil) controlled by a diaphragm (iris), a lens system (crystalline) and finally projecting over the retina where light sensitive vision cells or sensors (cones and rods) are located [4]. In this way the image is obtained by the imaging sensor and transforms the image in to numerical array. The array can be manipulated to enhance the visual quality, restoring degraded images, feature extraction for analysis and compression for faster transmission. In recent times, advanced computational methods of digital image processing have been proven effective in agricultural imaging applications [5]. The research from industrial applications could not be applied for agricultural applications directly because of the unpredictable nature of the environment as well as biological variations of objects [6]. In sustainable production, the role of technology in modern farming has shown promising outcomes. For the past decade there has been a rapid rise in image processing technology and other related technologies in agricultural applications. Imaging technology is being widely used in inspecting, monitoring, and grading a large class of agricultural and food products based on color, size/shape, and surface texture during postharvest handling, packing, and processing [7].Despite all the agricultural image processing applications, the source image must be free of unwanted features which might hinder further image processing.

Published By:

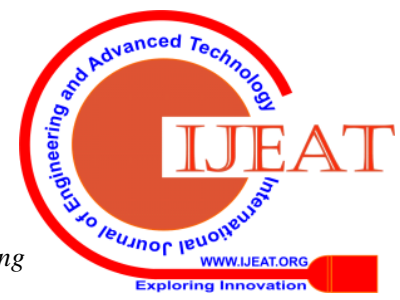


Hence image filtering is the key phase in agricultural image processing to ensure the quality of the image. This study mainly deals with the image denoising techniques based on wavelet transform.

\section{IMAGE DENOISING}

Image Denoising has been extensively studied area of digital image processing over the years and still poses challenges in finding the right approach. The presence of noise in all images is inevitable due to the influence of environment conditions during the image acquisition, compression for transmission and other factors which leads to feature distortion and loss of image information [8]. Noise obscures the necessary data for subsequent processing therefore reduced quality of the image. The purpose of image denoising is to remove noise while restoring the image information. Several methods have been proposed in image denoising, some focusing on spatial filtering which is a low pass frequency filter. In spatial filtering methods mean filter has been adopted on images corrupted by Gaussian noise but it smoothens the edges [9].Similar to mean filter, the Gaussian filter removes noise as well as edge details based on weighted average of the pixel values [10]. One method adopted to overcome the edge preserving problem has led to the median filtering which is widely used to remove impulse noise [11].Although it can achieve good results but it takes longer computational time. The image transform domain methods of cosine transform and wavelet transform domain methods evolved from Fourier transforms [8].However the Fourier transform gives only the frequency components of the signal.

\section{WAVELET DENOISING}

The Fourier Transform (FT) gives the frequency information of the signal but cannot provide at what time a particular frequency exist in the signal. To overcome the limitations of FT, Dennis Gabor adapted the Short Time Fourier Transform (STFT) to analyse only a section of the signal at a time which gives time-frequency components of the signal. This fixed window size technique gives good time resolution but poor frequency resolution for the narrow window and good frequency resolution but poor time resolution for the wide window [12]. The Wavelet Transform is similar to STFT but it analyses the signal in different scales.WT has the ability to analyse local details such as shift and discontinuities in the larger part of the signal. The WT gives time-space representation of the signal. The problem of continuous wavelet transform is that it analyse the signal by scaling and shifting continuously produces large number of coefficients. In 1976 Croiser, Esteban, and Galand proposed an effective technique to decompose the discrete time signals called discrete wavelet transform (DWT) [13]. The DWT analyse the signal based on powers of 2.The DWT can be efficiently implemented with high pass and low pass filters proposed by Mallat [14]. It decomposes the signal into approximation and detail coefficients where the former has the low frequency components and later has the high frequency components of the image. Further the image can be decomposed until an individual pixel left. The Agro Image De-noising (AID)

proposed in this study removes noise in agricultural images based on spline wavelet transform.

\section{PROPOSED DENOISING METHOD}

The block diagram of the proposed method is shown in the figure 1.The following steps show the methodology

1. The image with noise is decomposed by applying DWT.It is divided in to approximation coefficients and detail coefficients of vertical direction, horizontal direction and diagonal features of the image. The decomposition on image can be done upto $\mathrm{n}$ level. Here three level decomposition is applied.

2. At this stage,to denoise an image, a suitable thresholding to the wavelet coefficients is required. The intensity of the pixel is compared with the set thresholded value. If the pixel value is less than the threshold value the coefficients are suppressed to zero. The detail coefficients are thresholded with soft and hard thresholding methods to each decomposition level chosen.

3. Inverse DWT (IDWT) is performed on the obtained coefficients to bring back the desired image.

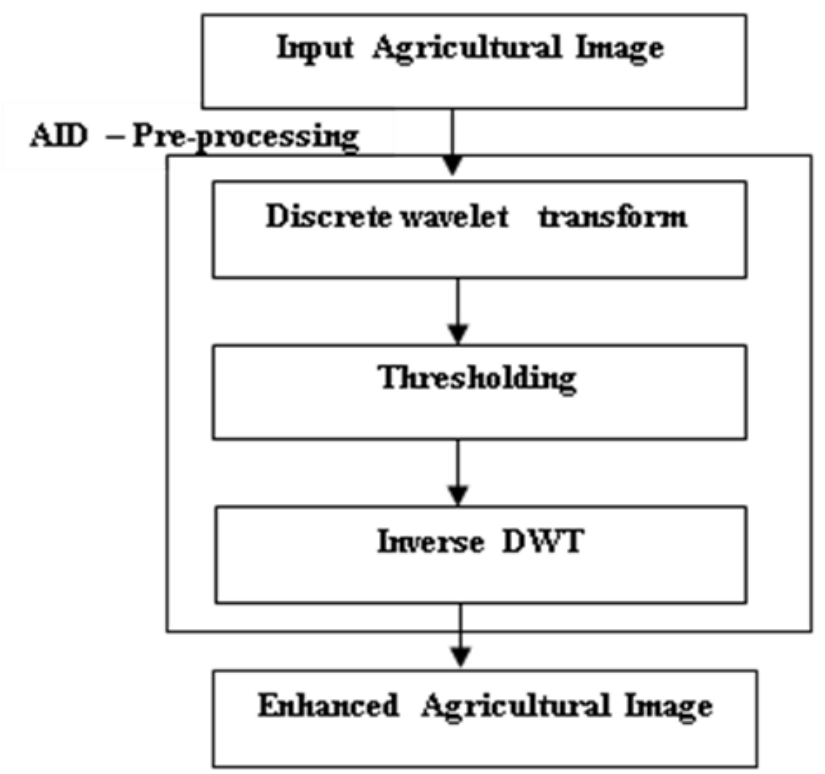

Fig.1. AID Architecture
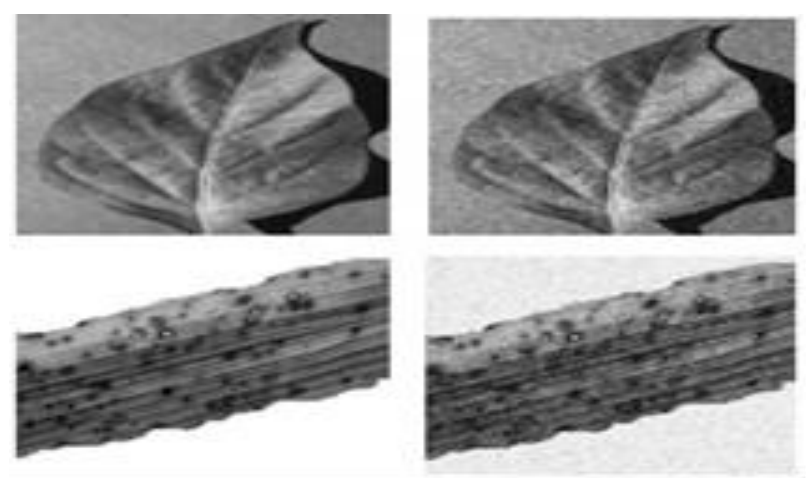

Fig.2. original and noisy image of Pepper leaf (Top row) Original and noisy image of Brown Spot Rice leaf (Bottom row) 


\section{A. Denoising Image using Fractional B Spline Scaling and Wavelets}

Splines are continuous polynomial piecewise function with pieces connected smoothly and the points where the pieces meet are called knots [15]. B-splines were utilized for signal reconstruction using polynomial equations [16].The Fractional B Splines have the fractional order of approximation and could be constructed from the fractional differences of one sided

function which is defined as [17].

$\beta^{\alpha}(x)=\frac{1}{\Gamma(\alpha+1)} \sum_{k=0}^{\infty}(-1)^{k}\left(\begin{array}{c}\alpha+1 \\ k\end{array}\right)(x-k)^{\alpha}$

Where ${ }^{\alpha}$ is the fractional power of the scaling function and $\Gamma(\alpha+1)$ is the Eulers gamma function defined as $\Gamma(p)=\int_{0}^{\infty} t^{p-1} e^{-t} d t$ for $p>0$. The fractional wavelet transform is constructed using Fourier expression. A fractional spline is the sum of shifted versions of the fractional b-spline [24]. The two scale relation is defined as

$\beta_{\tau}^{\alpha}\left(\frac{x}{2}\right)=\sum_{k} 2^{-\alpha}\left(\begin{array}{c}\alpha+1 \\ k\end{array}\right) \beta^{\alpha}(x-k)$

Table 1.SNR Values for Pepper Leaf Image and Brown Spot Rice Leaf image with Variance 0.04

\begin{tabular}{|c|c|c|c|}
\hline $\begin{array}{c}\text { Agricultural } \\
\text { Image }\end{array}$ & Wavelet Families & $\begin{array}{c}\text { Hard } \\
\text { Threshold }\end{array}$ & $\begin{array}{c}\text { Soft } \\
\text { Threshold }\end{array}$ \\
\hline \multirow{4}{*}{$\begin{array}{c}\text { Pepper Leaf } \\
\text { Image }\end{array}$} & haar( db1) & 38.89 & 28.94 \\
\cline { 2 - 4 } & orthogonal Spline & 33.96 & 28.93 \\
\cline { 2 - 4 } & b-spline & 53.09 & 38.93 \\
\cline { 2 - 4 } & dual spline & 26.54 & 22.93 \\
\hline \multirow{3}{*}{$\begin{array}{c}\text { Brown Spot } \\
\text { in rice Leaf } \\
\text { Image }\end{array}$} & orthogonal Spline & 35.71 & 30.88 \\
\cline { 2 - 4 } & b-spline & 48.3 & 39.94 \\
\cline { 2 - 4 } & dual spline & 26.75 & 23.66 \\
\cline { 2 - 4 } & \multicolumn{2}{|c}{} \\
\cline { 2 - 4 } & \multicolumn{2}{|c}{ on 1$)$} & 29.92 \\
\hline
\end{tabular}

Table 2.PSNR Values for Pepper Leaf Image and Brown Spot Rice Leaf image with Variance 0.04

\begin{tabular}{|c|c|c|c|}
\hline $\begin{array}{c}\text { Agricultural } \\
\text { Image }\end{array}$ & Wavelet Families & $\begin{array}{c}\text { Hard } \\
\text { Threshold }\end{array}$ & $\begin{array}{c}\text { Soft } \\
\text { Threshold }\end{array}$ \\
\hline \multirow{4}{*}{$\begin{array}{c}\text { Pepper Leaf } \\
\text { Image }\end{array}$} & Haar( db1) & 32.94 & 22.99 \\
\cline { 2 - 4 } & orthogonal Spline & 33.02 & 22.98 \\
\cline { 2 - 4 } & b-spline & 47.14 & 32.98 \\
\cline { 2 - 4 } & dual spline & 20.59 & 16.98 \\
\hline \multirow{3}{*}{$\begin{array}{c}\text { Brown Spot } \\
\text { in rice Leaf } \\
\text { Image }\end{array}$} & orthogonal Spline & 32.35 & 27.52 \\
\cline { 2 - 4 } & b-spline & 44.94 & 36.58 \\
\cline { 2 - 4 } & dual spline & 23.37 & 20.29 \\
\cline { 2 - 4 } & & & \\
\hline
\end{tabular}

\section{RESULTS AND DISCUSSION}

There are different types of wavelets are there in wavelet families. Some of the effective wavelets are haar (db1), orthogonal spline, b-spline and, dual spline. The four wavelets are used to evaluate Agricultural Images (Pepper Leaf Image and on Brown Spot in rice Leaf Image). Haar is simplest wavelet to implement and also known as Daubechies db1. Haar wavelet defines through branch of re-scaled square shaped wavelet putting together. Orthogonal spline wavelet used to construct through spline functions though are orthogonal. B-spline is the wavelet that mainly combination of spline wavelet. In wavelet, dual notation concept added because of, to introduce more flexibility for designing wavelet and dual spline wavelet used to do that The Fractional Wavelet transform package was used in MATLAB for agricultural image denoising proposed by [18]. The goal of this study is to find a suitable parameter to improve the noise corrupted agricultural images. The experiments have been implemented on two agricultural images taken from plant village and rice leaf diseases dataset. The images are of different size (256x256) the bell pepper leaf image and (200x200) the brown spot in rice leaf image. The original and Gaussian noise corrupted image is shown in fig 2. The wavelet families of spline and haar wavelets were used to generate the denoised image. The Additive Gaussian noise is added to the original image of variance 0.04 . The Fractional spline wavelet decomposition level of three is applied to test images. On each decomposition level, the detail coefficients are thresholded by hard threshold and soft threshold techniques to remove noise effectively and the performance of the thresholding methods are analysed. The results from Table 1 and 2 presents the SNR and PSNR measures of different spline wavelet families.
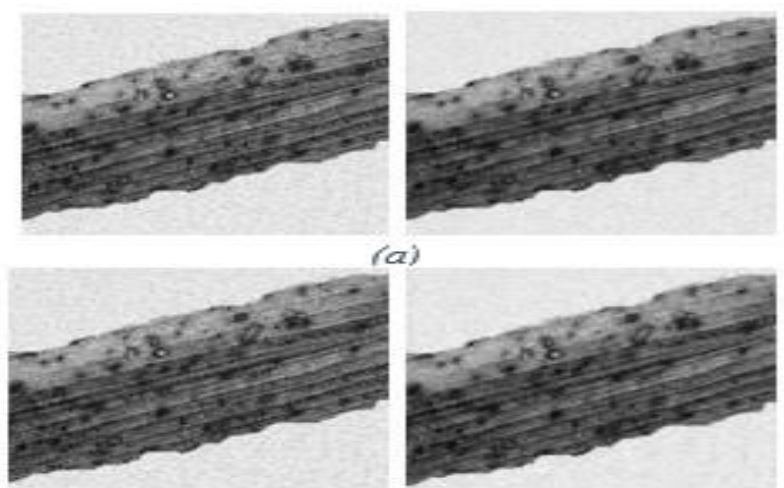

(a)
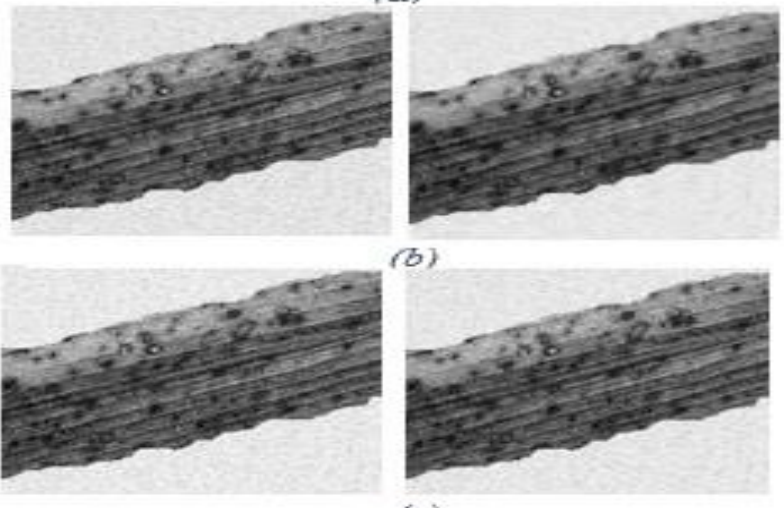

Fig.3. comparing the results of Brownspot Rice leaf denoised image using Hard Threshold (left column), Soft Threshold (right column) a.haar wavelet b. orthogonal spline wavelet c. b-spline wavelet 
The noise free image is reconstructed using inverse wavelet transform. The quality of the denoised image is assessed by peak signal to noise ratio (PSNR) and the equation is

$$
P S N R=10 . \log _{10}\left[\frac{255^{2}}{M S E}\right]
$$

Where the PSNR measures the ratio between the maximum intensity value of the image and the power of the noise in the image. The signal to noise ratio (SNR) measures ratio of the power of the signal to the power of noise and is calculated as

$$
S N R=\frac{\sum_{x=0}^{m-1} \quad \sum_{y=0}^{n-1} \quad \hat{f}(x, y)^{2}}{\sum_{x=0}^{m-1} \sum_{y=0}^{n-1}[f(x, y)-\hat{f}(x, y)]^{2}}
$$

Where the $f(x, y)$ is the original image and $\hat{f}(x, y)$ is the noisy image. Table $1-2$ present the PSNR and SNR measures with various wavelet threshold methods and different spline wavelet families.

The wavelet family of fractional spline wavelet transform is used to generate denoised images. According to the results display that the higher values of PSNR and SNR for b-spline semi orthogonal wavelet in hard threshold and soft threshold shows that the semiorthogonal spline wavelets is effective in denoising agricultural images. It can be seen from fig 3 and 4 the b-spline wavelet transform is better denoising compared to other wavelet families.
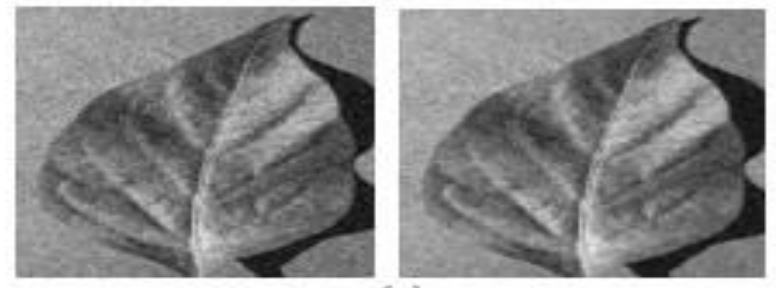

(a)
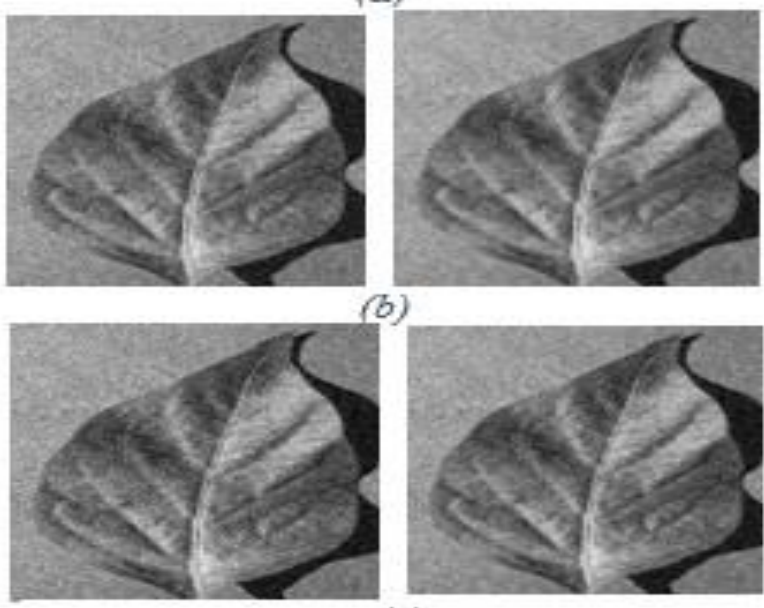

(c)

Fig.4. comparing the results of Pepper leaf denoised image using Hard Threshold (left column), Soft

Threshold (right column) a.haar wavelet b. orthogonal spline wavelet c. b-spline wavelet

In the pepper leaf image, in terms of SNR and PSNR, b-spline wavelet perform better for hard threshold and soft threshold than other wavelet and can be seen from fig 5 and 6 respectively. In Brown Spot rice Leaf Image, in terms of SNR and PSNR b-spline wavelet perform better for hard threshold and soft threshold than other wavelet and it is shown in fig 7 and fig 8 respectively. The results conclude that among the four wavelets haar (db1), orthogonal spline, b-spline and, dual spline, b-spline wavelet produce best results.

Hard Threshold Algorithm

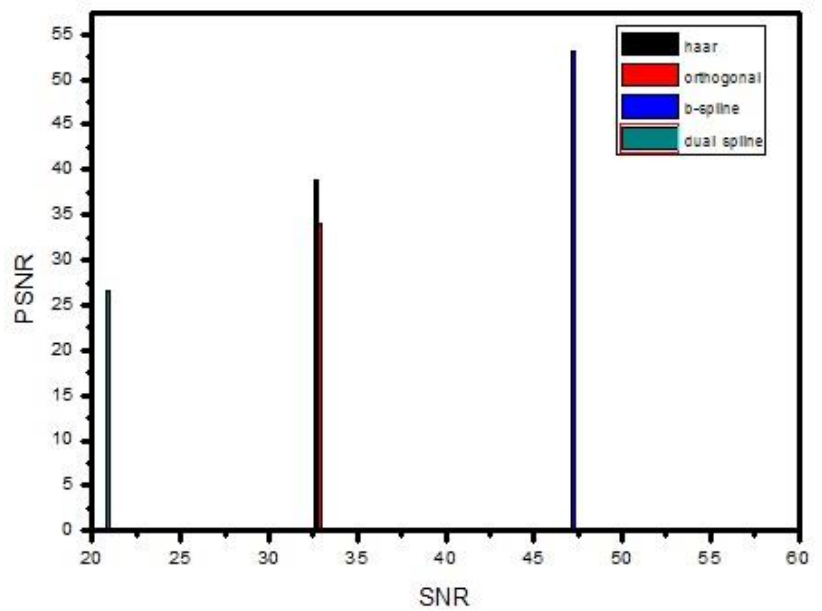

Fig.5. Comparison between SNR and PSNR values of denoised Pepper leaf image by various wavelets using hard threshold method

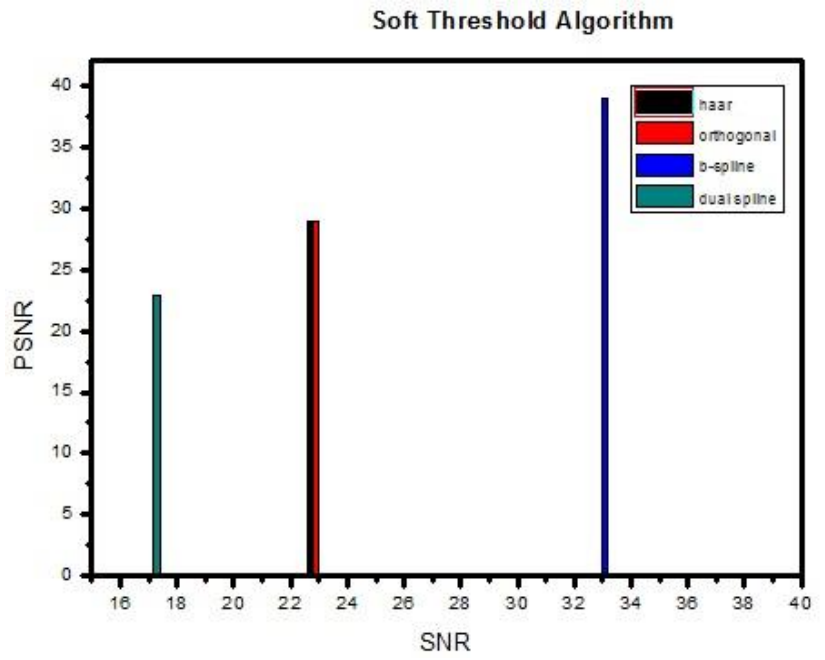

Fig.6. Comparison between SNR and PSNR values of denoised Pepper leaf image by various wavelets using soft threshold method

\section{CONCLUSION}

Imaging technology have been widely used in agricultural fields for identify and control disease in agricultural crops. This study analyses the wavelet family of spline wavelets to denoise noisy agricultural image. The proposed method provides effective results in denoising agricultural image. Here different wavelet families such as haar and spline wavelet families with various thresholding methods are applied. The hard threshold preserves the sharp details and the soft threshold suppress noise well. The quantitative parametric measures of the proposed approach for b-spline wavelets shows improvement in PSNR and SNR values. The fractional b-spline wavelet improves the quality of the image.

Published By:

Blue Eyes Intelligence Engineering \& Sciences Publication 


\section{ACKNOWLEDGMENT}

This research work has been written with the financial support of Rashtriya Uchchatar Shiksha Abhiyan (RUSAPhase 2.0) grant sanctioned vide Letter No. F.24-51/2014-U, Policy (TNMulti-Gen), Dept. of Edn. Govt. of India, Dt. 09.10.2018.

Hard Threshold Algorithm

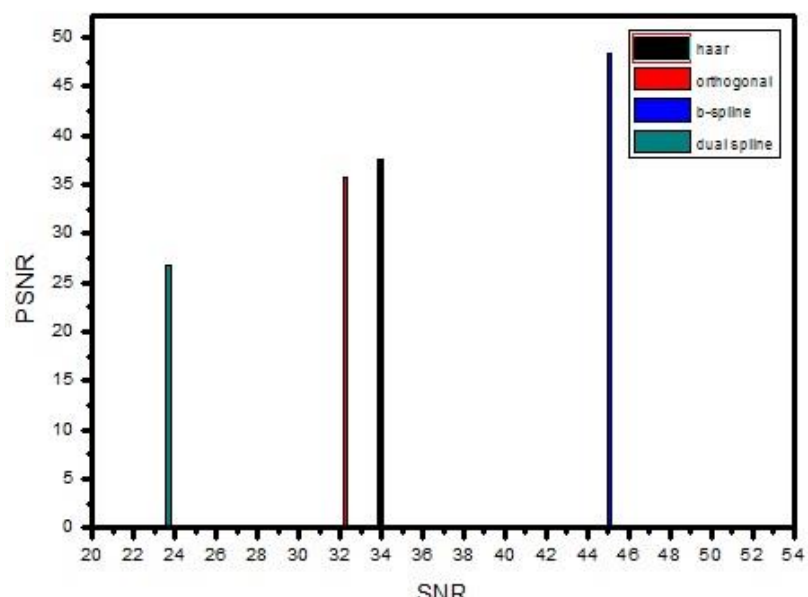

Fig.7. Comparison between SNR and PSNR values of denoised Brownspot Leaf image by various wavelets using hard threshold method

Soft Thres hold Algorithm

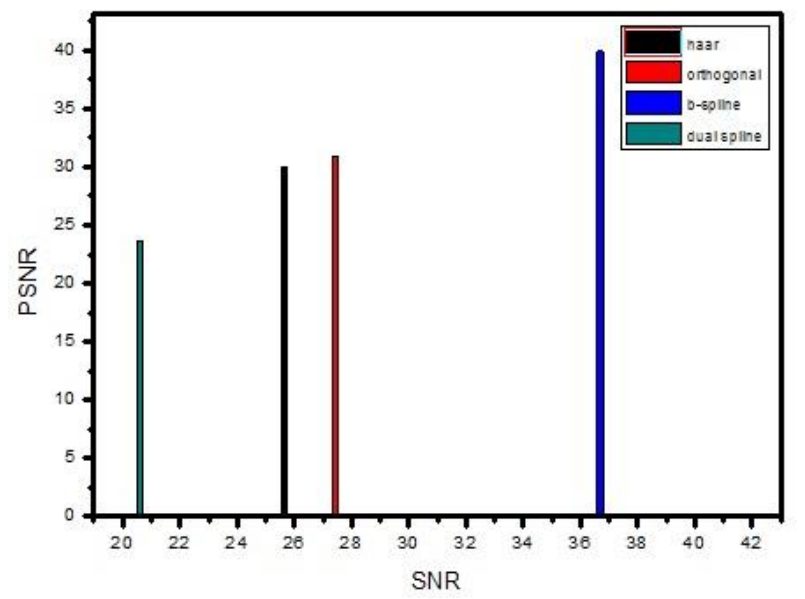

Fig.8. Comparison between SNR and PSNR values of denoised Brownspot Leaf image by various waveletsusing soft threshold method

\section{REFERENCES}

1. Md. Wasim Aktar, D. S. (2009, March). Impact of pesticides use in agriculture: their benefits and hazards. Interdiscip Toxicol, 1-12. doi:10.2478/v10102-009-0001-7

2. P.C. Abhilash, N. S. (2008). Pesticide use and application: An Indian scenario. Journal of Hazardous Materials, 165(1-3), 1-12.

3. Anuradha Badage, A. C. (2019). Farmer Advisory: A Crop Disease Detection System. International Research Journal of Engineering and Technology, 06(05), 770-775.

4. Adur Lagunas, O. D.-C.-R. (2017). Human Eye Visual Hyperacuity: A New. IEEE_Sensors_Journal, 1-8.

5. Carlos S. Pereira, R. M. (2017). Recent Advances in Image Processing Techniques for Automated Harvesting Purposes: A Review. Intelligent Systems Conference (pp. 566-575). London: IEEE.

6. Tillett, R. D. (1991). Image Analysis for Agricultural Processes: a Review of Potential Opportunities. J. agric. Engng Res., 247-258

7. Bosoon Park, R. L. (2015). Hyperspectral Imaging Technology in Food and Agriculture. Springer.

8. Linwei Fan, F. Z. (2019). Brief review of image denoising techniques. Visual Computing for Industry, Biomedicine, and Art, 1-12.

9. Pawan Patidar, S. S. (2010). Image De-noising by Various Filters for Different Noise. International Journal of Computer Applications, 45-50.

10. Manyu Wang, S. Z. (2014). A new image denoising method based on Gaussian filter. IEEE, 163-167.

11. Rui Ha1, P. L. (2016). An Improved Adaptive Median Filter Algorithm and Its Application. Advances in Intelligent Information Hiding and Multimedia Signal Processing (pp. 179-186). Springer.

12. Patil, R. (2015). Noise Reduction using Wavelet Transform and Singular. Eleventh International Multi-Conference on Information Processing-2015 (IMCIP-2015) (pp. 849-853). Procedia Computer Science.

13. Nadir Mustafa, J. P. (2015). Different, Medical Image De-Noising Schemes Using Different Wavelet Threshold Techniques. International Journal of Advanced Computer Science and Applications, 59-63.

14. S.G.Mallat. (1989). A Theory for Multiresolution Signal Decomposition: The Wavelet Representation. IEEE Transactions on Pattern Analysis and Machine Intelligence, 11, 674-693.

15. Unser, M. (1999). Splines A Perfect Fir for Signal and Image Processing IEEE Signal Processing Magazine, 22-38.

16. Fahmy, M. F. (2009). B-spline wavelets for signal denoising and image compression. Springer, 141-153.

17. Michael Unser, T. B. (2000). Fractional Splines and Wavelets. Society for Industrial and Applied Mathematics, 43-67.

18. T. Blu, M. U. (2000). The Fractional Spline Wavelet Transform: Definition and Implementation. Proceedings of the Twenty-Fifth IEEE International Conference on Acoustics, Speech, and Signal Processing (ICASSP'00) (pp 512-515). Istanbul: IEEE.

\section{AUTHORS PROFILE}

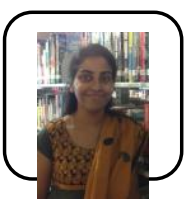

Sangeetha Muthiah is pursuing $\mathrm{PhD}$ at the department of Computational Logistics, Alagappa University, Karaikudi. She is currently doing research in the area of image enhancement. Her research interest includes image processing, deep learning.

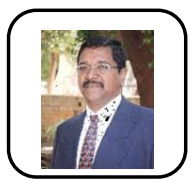

Prof. A. Senthilrajan has been working as the Director of Management Information System (MIS) at Alagappa University, Karaikudi.He has completed his PhD. (Computer Sciennce) in Alagappa University. He has attended many national and international seminars, conferences and presented research papers and also published research articles in national and international journals. He is member of IEEE and his area of research specialization is Digital Image Enhancement, Artificial neural networks, Network Security.

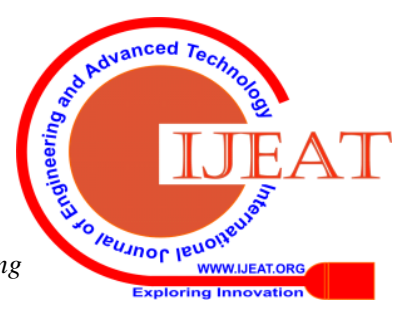

